\title{
Upregulation of microRNA-520a-3p inhibits the proliferation, migration and invasion via spindle and kinetochore associated 2 in gastric cancer
}

\author{
HUI SU ${ }^{1}$, FENG REN ${ }^{1}$, HAITAO JIANG ${ }^{1}$, YUNJIE CHEN ${ }^{1}$ and XIAOXIANG FAN ${ }^{2}$ \\ Departments of ${ }^{1}$ General Surgery and ${ }^{2}$ Interventional Therapy, Ningbo No. 2 Hospital, Zhejiang 315010, P.R. China
}

Received September 30, 2018; Accepted June 5, 2019

DOI: $10.3892 / 01.2019 .10663$

\begin{abstract}
MicroRNAs (miR) serve important roles in the development and progression of tumors by targeting different genes. miR-520a-3p reported in lung and breast cancers as a tumor suppressor gene. However, the expression and functional significance of miR-520a-3p is not completely understood in gastric cancer (GC). In the present study, it was demonstrated that the expression levels of miR-520a-3p were significantly downregulated in GC tissues and cells using RT-qPCR. In addition, downregulated expression of miR-520a-3p was associated with the clinical stage of the tumor and invasion in patients with GC. Furthermore, overexpression of miR-520a-3p significantly inhibited cell proliferation, invasion and migration in SGC-7901 and MGC-803 GC cell lines using proliferation, wound healing and cell invasion assays. Spindle and kinetochore associated 2 (SKA2) was upregulated in GC cells using western blot analysis and a target gene of miR-520a-3p; miR-520a-3p mimics significantly reduced SKA2 expression. In addition, upregulation of SKA2 protein expression SKA2 reversed the miR-520a-3p-mediated inhibition of SGC-7901 cell proliferation, migration and invasion. In conclusion, miR-520a-3p functioned as a tumor suppressor gene by targeting SKA2 in GC cell lines, and may serve as a novel prognostic and potential therapeutic marker.
\end{abstract}

\section{Introduction}

Gastric cancer (GC) is a common cancer worldwide and remains the third leading cause of cancer-related death, following lung and liver cancer, accounting for 1.3 million cases of GC and 819,000 GC-associated cases of mortality

Correspondence to: Dr Xiaoxiang Fan, Department of Interventional Therapy, Ningbo No. 2 Hospital, 41 Northwest Street, Ningbo, Zhejiang 315010, P.R. China

E-mail: fanxiaoxiang163@163.com

Key words: microRNA-520a-3p, spindle and kinetochore-associated protein 2, proliferation, migration, invasion, gastric cancer in 2015 (1). The high incidence and mortality rates of GC have been demonstrated throughout the developed world, and GC adversely affects patients' health and quality of life (2). Although notable progress has been achieved in treating patients with GC based on advances in technology $(3,4)$, the precise molecular mechanisms underlying GC development and progression remain unknown, and require further investigation.

MicroRNAs (miRNAs/miRs) can function as oncogenes or tumor suppressor genes, and regulate gene expression through translational repression or mRNA degradation by binding to the 3'-untranslated region (3'UTR) of their target mRNAs $(5,6)$. Previous studies have identified that miRNAs exhibit aberrant expression and serve essential roles in GC (7-10). Therefore, further investigation into the expression and function of miRNAs may improve understanding of the molecular mechanisms responsible for the pathogenesis of GC.

Previous studies have demonstrated that the expression levels of miR-520a-3p are downregulated in non-small cell lung cancer cells. Decreased levels of miR-520a-3p expression were associated with poorer overall survival (11). Additionally, the upregulation of miR-520a-3p significantly suppressed the proliferation, cell cycle progression and metastatic activity by targeting mitogen-activated protein kinase kinasekinase2, HOXD8 or PI3K/AKT/mTOR $(12,13)$. miR-520a-3p was also shown to inhibit proliferation and metastatic activity in breast cancer through cyclinD1, and in colorectal cancer via epidermal growth factor receptor $(14,15)$. In osteosarcoma, dexmedetomidine has been reported to upregulate miR-520-3p, which directly targeted AKT1 to inhibit MG63 cell proliferation and migration and promote apoptosis. In addition, LINC01116 has been reported to target miR-520a-3p, which affectedinterleukin-6 receptor, thereby promoting proliferation and migration via the JAK-STAT signaling pathway $(16,17)$. However, the expression profile and biological significance of miR-520a-3p in GC are unknown at present, to the best of our knowledge.

The present study examined the expression of miR-520a-3p in GC tissues and cells and demonstrated the functions of miR-520a-3p overexpression in GC cell proliferation, invasion and migration. Together, these data demonstrate that miR-520a-3p may be a novel therapeutic target for the treatment of GC. 


\section{Materials and methods}

Patients. GC specimens and adjacent tissues were received from patients who were diagnosed with GC and underwent surgery at Ningbo No. 2 Hospital (Zhejiang, China) between August 2016 and May 2017. A total of 80 female patients,

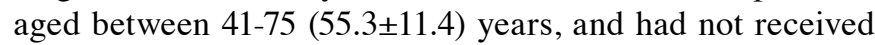
local or systemic therapy prior to surgery at Ningbo No. 2 Hospital. The present study was approved by The Research Ethics Committee of Ningbo No. 2 Hospital, and all subjects provided the information written consent. The samples were graded according to the 8th edition of the AJCC staging classification system (18). The relationship between miR-520a-3p levels and the clinical characteristics of enrolled patients were analyzed.

Cell culture. All GC cell lines (SGC-7901, BGC-823, and MGC-803) and a normal gastric epithelial cell line (GES-1) used in the present study were obtained from the Institute of Biochemistry and Cell Biology (Shanghai, China) and maintained in DMEM (HyClone; GE Healthcare Life Sciences) medium (SGC-7901, BGC-823) or RPMI 1640 (HyClone; GE Healthcare Life Sciences) medium (MGC-803, GES-1) containing $10 \%$ FBS (Shanghai Ex CellBiology, Inc.) at $37^{\circ} \mathrm{C}$, in a humidified incubator with $5 \% \mathrm{CO}_{2}(\mathrm{Memmert} \mathrm{GmbH})$.

Cell transfection. Cells (SGC-7901 and MGC803) were transfected with miR-520a-3p mimics (100 pmol; forward, 5'-AAA GUGCUUCCCUUUGGACUGU-3' and reverse, 5'-AGU CCAAAGGGAAGCACUUUUU-3') or miR-520a-3p negative control (NC; 100 pmol; forward, 5'-UUCUCCGAACGU GUCACGUTT-3' and reverse, 5'-ACGUGACACGUUCGG AGAATT-3'; both from Shanghai GenePharma Co. Ltd.), or spindle and kinetochore associated 2 (SKA2) cDNA plasmid (pLenti-SKA2-Puro) or NC (pLenti-C-Myc-DDK-P2A-Puro) (4 $\mu \mathrm{g}$; both from OriGene Technologies, Inc.) using Lipofectamine $^{\mathrm{TM}} 2000$ transfection reagent (Invitrogen; Thermo Fisher Scientific, Inc.) following the manufacturer's protocol. Cells were incubated with the transfection reagent for $24 \mathrm{~h}$, following which RNA and protein were extracted. No treatment or transfection was performed in the MOCK group.

The SKA2 cDNA plasmid (without the $3^{\prime} \mathrm{UTR}$ ) and miR-520a-3p mimics were co-transfected into SGC-7901 cells, which subsequently underwent western blot analyses, cell proliferation assays, wound healing assay and cell invasion assays.

Western blotting. Total cell protein was extracted by 1XSDS (Sigma-Aldrich; Merck KGaA) and quantified by a bicinchoninic acid assay (Beyotime Institute of Biotechnology). Cellular proteins $(40-50 \mu \mathrm{g})$ were loaded onto a $12 \%$ polyacrylamide gel and resolved by SDS-PAGE. After separation, the proteins were transferred onto polyvinylidene difluoride membranes (EMD Millipore) for immunoblotting. Membranes were blocked with 5\% BSA (Beijing Solarbio Science \& Technology Co., Ltd.) for $2 \mathrm{~h}$ at room temperature, the membranes were incubated with primary antibodies overnight at $4{ }^{\circ} \mathrm{C}$. The primary antibodies used were Ki-67 (1:1,500; Abcam; cat no. ab15580), matrix metalloproteinase (MMP)-2 (1:1,500; Abcam; cat no. ab7033), MMP-9 (1:1,500; Abcam; cat no. ab137651), SKA2 (1:1,500;
Abcam; cat no. ab91551) and GAPDH (1:2,000; Cell Signaling Technology, Inc.; cat. no. 5174). The membranes were subsequently incubated for $1 \mathrm{~h}$ at room temperature with goat anti-rabbit IgG-horseradish peroxidase (HRP) or goat anti-mouse IgG-HRP (1:5,000; Wuhan Boster Biological Technology, Ltd.; cat. nos. BA1054 and BA1050, respectively). The protein signals on the membrane were detected using an ECL reagent (Absin Biotechnology Co., Ltd.) and visualized using a chemiluminescence imaging system (LI-COR Biosciences).

$R T-q P C R$. The expression levels of miR-520a-3p and SKA2 were determined using RT-qPCR. Total RNA was extracted using TRIzol ${ }^{\circledR}$ reagent (Invitrogen; Thermo Fisher Scientific, Inc.) according to the manufacturer's protocol. The RNA was reverse transcribed to cDNA using a TaqMan reverse transcription kit (Thermo Fisher Scientific, Inc.) with the following RT protocol; $15 \mathrm{~min}$ at $37^{\circ} \mathrm{C}$ and $5 \mathrm{sec}$ at $85^{\circ} \mathrm{C}$. cDNA was amplified using SYBR-Green PCR Master mix (Roche Diagnostics) on a LightCycler 480 system. The thermocycling conditions were as follows: $95^{\circ} \mathrm{C}$ for $10 \mathrm{~min}$; and followed by 45 cycles, $95^{\circ} \mathrm{C}$ for $10 \mathrm{sec}$ and $60^{\circ} \mathrm{C}$ for $60 \mathrm{sec}$. Fold changes were calculated by relative quantification $\left(2^{-\Delta \Delta \mathrm{Cq}}\right)$ using endogenous U6 and GAPDH as references for miR-520a-3p and SKA2 expression, respectively (19). The primers used for miR-520a-3p were: Forward, 5'-ACACTCCAGCTGGGA AAGTGCTTCCC-3' and reverse, 5'-CTCAACTGGTGTCGT GGA-3'. For U6, the primers used were: Forward, 5'-CTC GCTTCGGCAGCACA-3' and reverse, 5'-AACGCTTCACGA ATTTGCGT-3'. For SKA2, the primers used were: Forward, 5'-CTGAAACTATGCTAAGTGGGGGAG-3' and reverse, 5'-TTCCAAACATCCTGACACTCAAAAG-3'. For GAPDH, the primers used were: Forward, 5'-AAGCCTGCCGGTGAC TAAC-3' and reverse, 5'-GCATCACCCGGAGGAGAAAT-3'.

Cell proliferation assay. miR-520a-3p mimics, SKA2 cDNA and negative control-transfected GC cells (SGC-7901 and MGC803) were seeded in 96-well plates ( $1 \times 10^{4}$ cells/well). A total of $20 \mu \mathrm{l}$ Cell Titer $96^{\circledR}$ AQueous One Solution (Promega Corporation) was added to each well to determine cell viability at 4, 24, 48, 72 and $96 \mathrm{~h}$ after seeding. Cells were incubated at $37^{\circ} \mathrm{C}$, in a humidified incubator with $5 \% \mathrm{CO}_{2}$ for $3 \mathrm{~h}$. Subsequently the absorbance of each well was measured at $490 \mathrm{~nm}$ on a spectrophotometer (Beckman Coulter, Inc.).

Wound healing assay. Cells were cultured in 6-well plates $\left(1 \times 10^{5}\right.$ cells/well $)$ for $24 \mathrm{~h}$. Once the cellular density had reached $\sim 100 \%$, a vertical line was scraped in the culture using a 1-ml pipette tip. The cells were rinsed with PBS and incubated with fresh serum-free medium at $37^{\circ} \mathrm{C}$. The widths of the gap at 0 and $24 \mathrm{~h}$ were compared to assess the distance of migration using fluorescence microscopy.

Cell invasion assays. Cell invasion assays were performed using a Matrigel invasion chamber (24-well plates; $8-\mu \mathrm{m}$ pore size; Corning Inc.) according to the manufacturer's protocol. A total of $5 \times 10^{4}$ cells were seeded in the upper chambers of the wells in $100 \mu \mathrm{l}$ FBS-free medium, and the lower chambers contained DMEM supplemented with $20 \%$ FBS. The cells were incubated for $24 \mathrm{~h}$ at $37^{\circ} \mathrm{C}$, after which the cells on the 
Table I. Association between miR-520a-3p expression and clinicopathological factors in 80 primary gastric cancer tissues.

\begin{tabular}{|c|c|c|c|c|}
\hline \multirow[b]{2}{*}{ Characteristic } & \multirow[b]{2}{*}{ Patients, $\mathrm{n}$} & \multicolumn{2}{|c|}{ miR-520a-3p expression level } & \multirow[b]{2}{*}{ P-value } \\
\hline & & Low, n (\%) & High, n (\%) & \\
\hline Age, years & & & & 0.990 \\
\hline$\leq 50$ & 38 & $28(73.7)$ & $10(26.3)$ & \\
\hline$>50$ & 42 & $31(73.8)$ & $11(26.2)$ & \\
\hline Tumor diameter, $\mathrm{cm}$ & & & & 0.067 \\
\hline$\leq 3$ & 24 & $21(87.5)$ & $3(12.5)$ & \\
\hline$>3$ & 56 & $38(67.9)$ & $18(32.1)$ & \\
\hline Tumor TNM staging & & & & $0.016^{\mathrm{a}}$ \\
\hline $\mathrm{I}+\mathrm{II}$ & 43 & $27(62.8)$ & $16(37.2)$ & \\
\hline III+IV & 37 & $32(86.5)$ & $5(13.5)$ & \\
\hline Tumor histological overall NHS grade & & & & 0.305 \\
\hline 1 & 7 & $4(57.1)$ & $3(42.9)$ & \\
\hline 2 & 41 & $33(80.5)$ & $8(19.5)$ & \\
\hline 3 & 32 & $22(68.8)$ & $10(31.3)$ & \\
\hline Invasion & & & & $0.001^{\mathrm{b}}$ \\
\hline $\mathrm{T} 1+\mathrm{T} 2$ & 27 & $14(51.9)$ & $13(48.1)$ & \\
\hline $\mathrm{T} 3+\mathrm{T} 4$ & 53 & $45(84.9)$ & $8(15.1)$ & \\
\hline Pathological type & & & & 0.412 \\
\hline Adenocarcinoma & 69 & $52(75.4)$ & $17(24.6)$ & \\
\hline Non-adenocarcinoma & 11 & $7(63.6)$ & $4(36.4)$ & \\
\hline
\end{tabular}

${ }^{\mathrm{a}} \mathrm{P}<0.05,{ }^{\mathrm{b}} \mathrm{P}=0.001$. miR, microRNA; TNM, Tumor-Node-Metastasis; NHS, National Health Service.

filter surface were stained with $0.1 \%$ crystal violet for $30 \mathrm{~min}$ at room temperature and images were captured with an inverted fluorescence microscope (Nikon Corporation), representative images were captured at x100 magnification. The absorbance was measured at $590 \mathrm{~nm}$.

Bioinformatics. Potential miR-520a-3p targets were predicted and analyzed using the following two publicly available databases: TargetScanv7.2 (http://www.targetscan.org), and miRanda (http://www.microrna.org/microrna/getGeneForm.do).

Luciferase assay. The predicted miR-520a-3p binding sites on the 3'UTR of wild-type (WT) SKA2 (MirTarget-SKA2-3U-WT), together with a corresponding mutant (mut) miR-520a-3p binding sites on the 3'UTR of SAK2 (MirTarget-SKA2-3U-Mut), were synthesized and transfected into the pGL3 vector (Promega Corporation). SGC-7901 cells were seeded in a 24 -well plates $\left(5 \times 10^{4}\right.$ cells/well $)$, the WT or Mut 3'UTR vectors, and miR-520a-3p mimics or NC were co-transfected using Lipofectamine ${ }^{\mathrm{TM}}$ 2000. The relative luciferase activity was measured after $48 \mathrm{~h}$ using a Dual-Luciferase Reporter assay system according to the manufacturer's protocols (Promega Corporation). Luciferase activity was normalized to Renilla luciferase activity.

Statistical analysis. All experiments were repeated three times and data are presented as the mean \pm standard deviation. One-way ANOVA and Fisher's least significant difference tests were used to calculate P-values between multiple groups. Data in Table I were analyzed using $\chi^{2}$ tests of four-fold table. Statistical analyses were performed using SPSS 15.0 software (SPSS, Inc.). $\mathrm{P}<0.05$ was considered to indicate a statistically significant difference.

\section{Results}

miR-520a-3p expression levels are downregulated in $G C$ and are associated with clinical stage and tumor invasion. miR-520a-3p expression levels in 80 GC samples and adjacent tissue samples were measured by RT-qPCR, and the levels were normalized to U6. The results demonstrated that miR-520a-3p expression levels were significantly decreased in GC tissues compared with the adjacent normal tissues $(\mathrm{P}<0.01$; Fig. 1A). Furthermore, the expression levels of miR-520a-3p in three human GC cell lines (SGC-7901, BGC-823 and MGC-803) were evaluated and compared with the normal gastric epithelial cell line (GES-1). miR-520a-3p expression was significantly upregulated in the GES-1 compared with the other three cell lines (Fig. 1B).

To investigate the clinical significance of miR-520a-3p in patients with GC, miR-520a-3p expression in a cohort of 80 specimens was analyzed (Table I). The miR-520a-3p levels were significantly associated with clinical stage $(\mathrm{P}<0.05)$ and tumor invasion $(\mathrm{P}<0.001)$ in $\mathrm{GC}$ tissues; however, they were not associated with patient age, tumor size, histological grade or pathological type $(\mathrm{P}>0.05)$. 

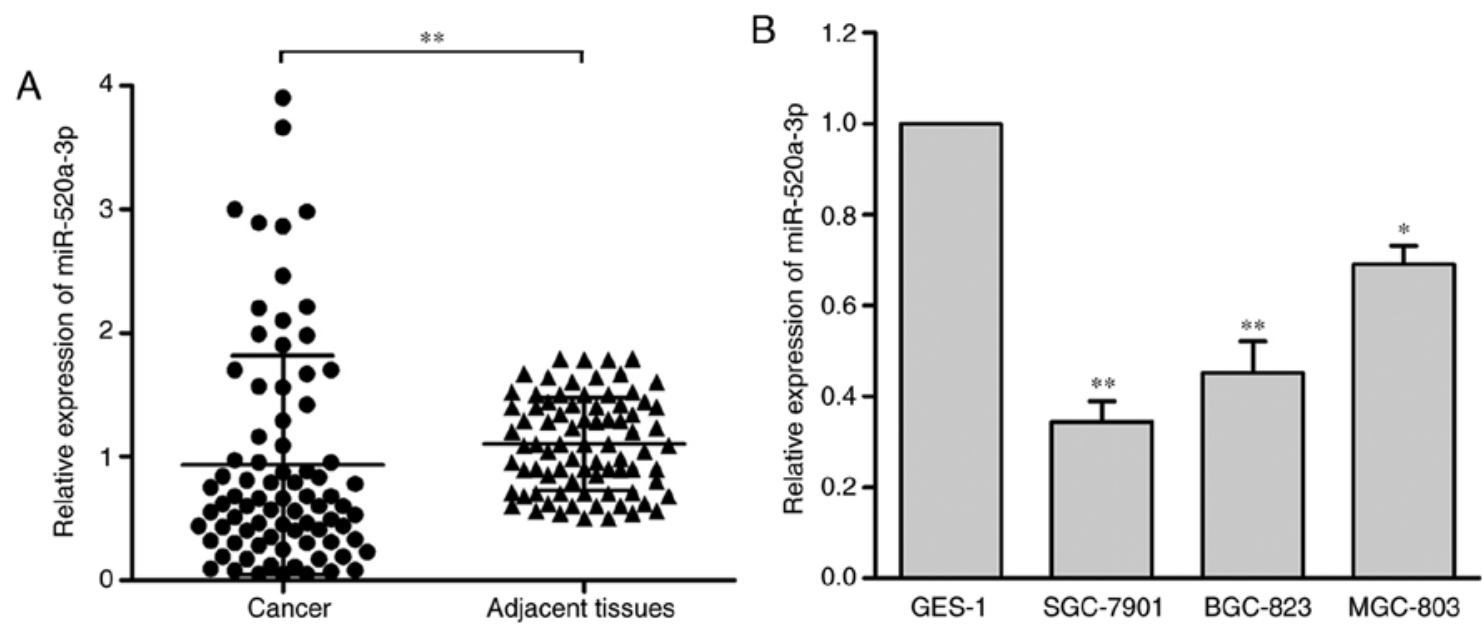

Figure 1. miR-520a-3p expression is decreased in GC tissues and cell lines. (A) miR-520a-3p expression was detected via reverse transcription-quantitative PCR analysis in $80 \mathrm{GC}$ tissues compared with adjacent non-tumor tissues. ${ }^{* *} \mathrm{P}<0.01$ vs. adjacent non-tumor tissues (B) miR-520a-3p expression was analyzed in three GC cell lines (SGC-7901, BGC-823, and MGC-803) compared with in a normal gastric epithelial cell line (GES-1). The levels of miR-520a-3p expression were normalized to U6. ${ }^{*} \mathrm{P}<0.05,{ }^{* *} \mathrm{P}<0.01$ vs. GES-1. miR, microRNA; GC, gastric cancer.

miR-520a-3p suppresses the proliferation of GC cells. To investigate the functional effects of miR-520a-3p expression in GC, SGC-7901 and MGC-803 cells were transfected with MOCK, miR-NC or miR-520a-3p mimics. Treatment with miR-520a-3p mimics significantly increased the expression levels of miR-520a-3p $(\mathrm{P}<0.001)$, compared with the MOCK group (Fig. 2A). Following the upregulation of miR-520a-3p expression, the effect on cell proliferation was examined using cell proliferation assay. Compared with the control cells, cells transfected with miR-520a-3p mimics demonstrated significantly reduced levels of proliferation (Fig. 2B). Recent studies have demonstrated that Ki-67 is closely associated with cell proliferation $(20,21)$. Investigating the levels of Ki-67 showed that miR-520a-3p mimics significantly decreased Ki-67 expression in GC cells ( $\mathrm{P}<0.01$; Fig. $2 \mathrm{C})$.

miR-520a-3p decreases the migration and invasion of GC cells. To examine how miR-520a-3p affects the migration and invasion of GC cells, the effects on cell migration and invasion were investigated in vitro using scratch wound healing and Transwell assays, respectively. The data demonstrated that transfection of cells with miR-520a-3p mimics significantly inhibited GC cell migration and invasion (Fig. 3A and B) compared with MOCK and NC groups.

Interactions between cell-surface proteins and the extracellular matrix (ECM) serve a critical role in tumor migration and invasion (22). As MMPs, particularly MMP-2 and MMP-9, digest ECM components, they are closely associated with the metastatic capabilities of cancerous cells (23). Transfection of miR-520a-3p mimics led to significantly decreased protein expression levels of MMP-2 and MMP-9 in GC cells compared with the MOCK and NC transfections (Fig. 3C).

miR-520a-3p directlytargets SKA2 in the SGC-7901GC cell line. To investigate the molecular mechanism underlying the effects of miR-520a-3p on GC cellular functions, SKA2 mRNA was identified as a potential miR-520a-3p target using bioinformatics (Fig. 4A). Subsequently, the WT or Mut target region sequence of the SKA2 3'UTR were cloned and inserted into a luciferase reporter vector (Fig. 4A). The luciferase reporter assays revealed that miR-520a-3p significantly decreased the luciferase activity in the reporter gene containing the WT 3'UTR ( $\mathrm{P}<0.01)$; however, no difference was observed with the Mut 3'UTR ( $P>0.05$; Fig. 4B). To demonstrate whether miR-520a-3p affected SKA2 expression in GC, expression of SKA2 in SGC-7901 cells transfected with miR-520a-3p mimics was measured. The data demonstrated that transfection with miR-520a-3p mimics significantly decreased SKA2 mRNA and protein expression levels, compared with the MOCK group ( $\mathrm{P}<0.01$; Fig. $4 \mathrm{C}$ and $\mathrm{D})$. Furthermore, the protein expression of SKA2 in SGC-7901, BGC-823, and MGC-803 was significantly in all $3 \mathrm{GC}$ cell lines compared with GES-1 (P<0.001; Fig. 4E).

Overexpression of SKA2 reverses the miR-520a-3p-mediated inhibition of SGC-7901 cell proliferation, migration and invasion. To determine whether SKA2 overexpression reversed the inhibitory effects of miR-520a-3p mimics on SGC-7901 cells, SKA2 cDNA without the 3'UTR and miR-520a-3p mimics were co-transfected into SGC-7901 cells $(\mathrm{P}<0.01$; Fig. 5A). Western blotting and cell viability assays showed that the decrease in SKA2 expression and cell viability following miR-520-3p overexpression was reversed when SKA2 was co-transfected with miR-520a-3p mimics (Fig. 5B and C). Migration and invasion assays demonstrated that SKA2 co-transfection significantly reversed the miR-520a-3p-mediated decrease in cell migration and invasion in SGC-7901 cells (Fig. 5D and E).

\section{Discussion}

miR-520a-3p serves an tumor suppressive role in certain types of cancer (11-17). However, its functions and expression in GC have not been studied, to the best of our knowledge.

The results demonstrated that miR-520a-3p was downregulated both in human GC tissues and GC cell lines compared with adjacent tissues and a normal gastric cell line. In addition, the expression of miR-520a-3p was associated with clinical stage and tumor invasion in patients with GC. Subsequent 
A

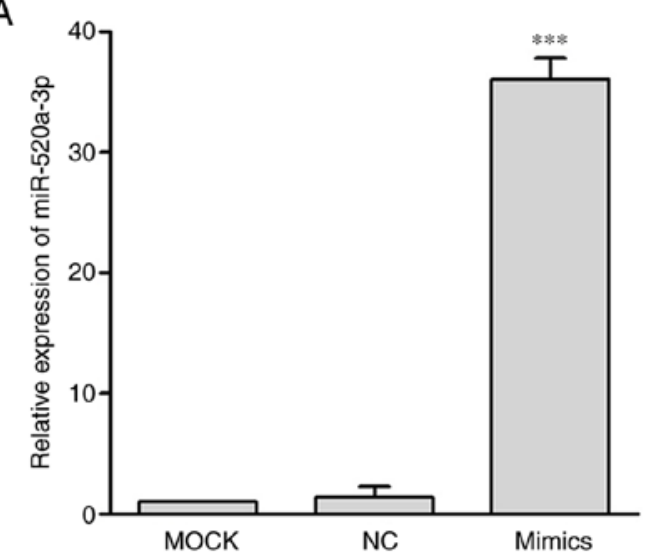

B

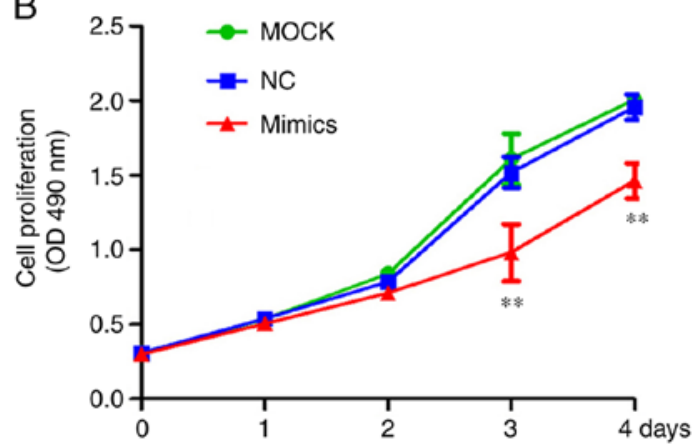

C
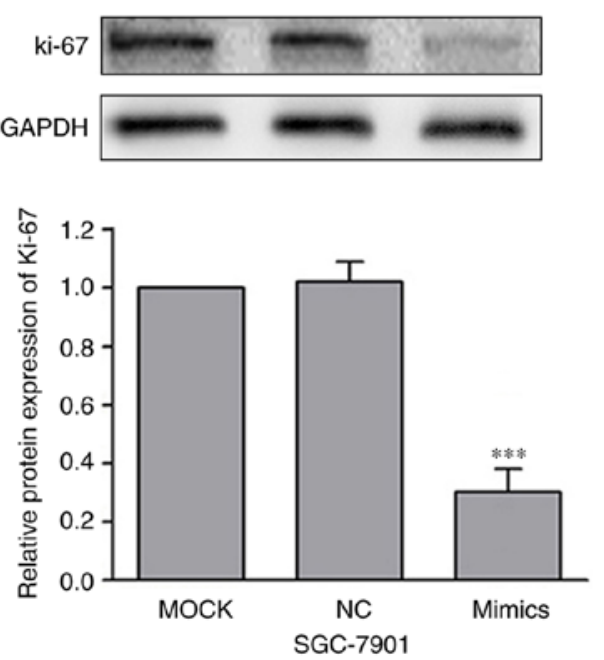
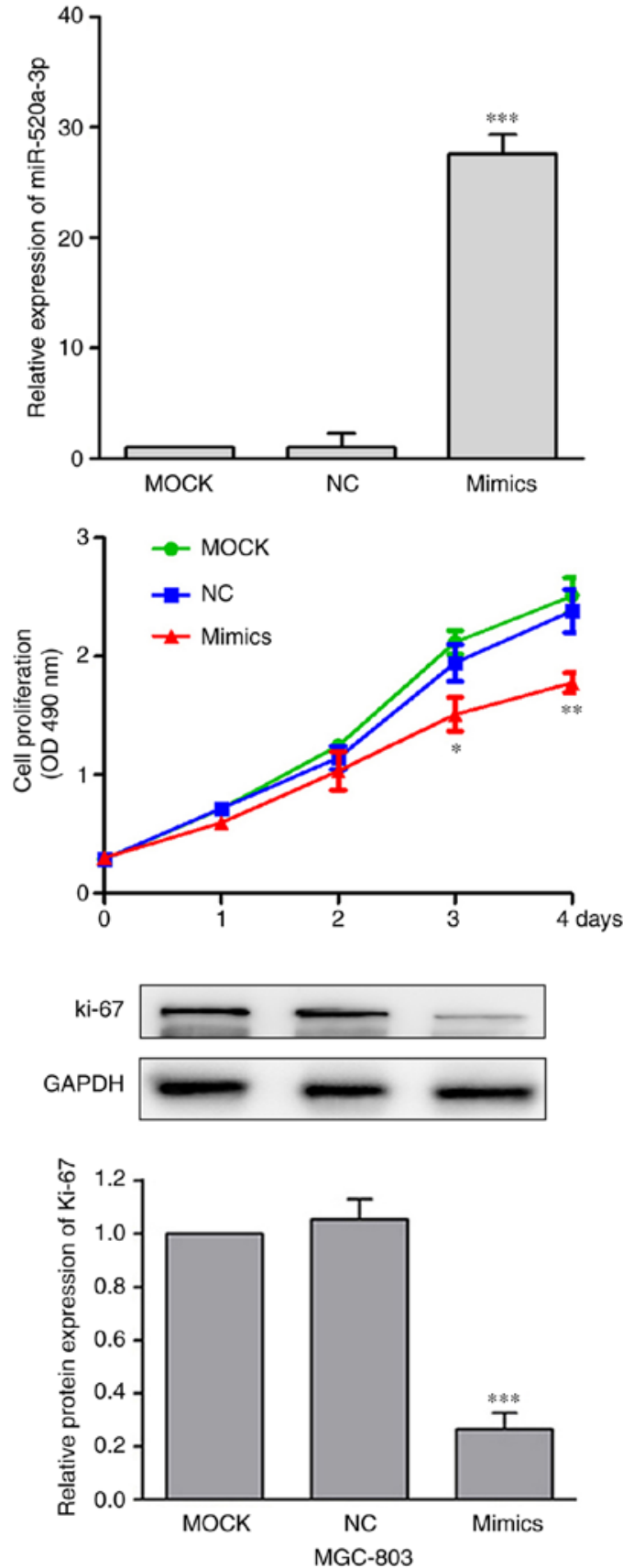

Figure 2. Overexpression of miR-520a-3p inhibits the proliferation of GC cells. (A) Following transfection with miR-520a-3p mimics, the mRNA expression levels of miR-520a-3p were significantly increased in SGC-7901 and MGC-803 cells compared with the respective MOCK and NC groups. ${ }^{* * *} \mathrm{P}<0.001$ vs. MOCK and NC. (B) GC cell viability in SGC-7901 and MGC-803 cells was examined by cell proliferation assay following the overexpression of miR-520a-3p. Transfection of the mimics significantly reduced proliferation. ${ }^{*} \mathrm{P}<0.05,{ }^{* *} \mathrm{P}<0.01$ vs. MOCK and NC. (C) Western blot of the Ki-67 expression levels normalized to GAPDH. ${ }^{* * *} \mathrm{P}<0.001$ vs. MOCK and NC. miR, microRNA; GC, gastric cancer; MOCK, mock transfected cells; NC, negative control; OD, optical density.

experimentation revealed that the upregulation of miR-520a-3p inhibited the proliferation, migration and invasion of $\mathrm{GC}$ cells (SGC-7901 and MGC-803), potentially by directly inhibiting SKA2. Furthermore, miR-520a-3p significantly inhibited the level of SKA2, and overexpression of SKA2 rescued the miR-520a-3p-mediated inhibition of SGC-7901 cell proliferation, migration and invasion. Therefore, the present study further confirmed the several biological functions attributed to miR-520a-3p, including the reduction in GC cell viability, and inhibition of cell migration and invasion following transfection with miR-520a-3p mimics, as well as the molecular mechanism underlying the miR-520a-3p-mediated inhibitory effect (11-17). SKA2 is located on chromosome 17 of the human genome and is a conserved protein involved in the kinetochore complex (24). SKA2, together with its cofactors SKA1 and SKA3, constitute the SKA complex, which maintains the metaphase plate and/or spindle checkpoint silencing $(25,26)$. Recently, SKA2 was also identified to exert a function in tumorigenesis. Aberrant expression patterns of SKA2 have been reported in lung cancer (27-29), breast cancer (30-32), osteosarcoma $(33,34)$ and kidney cancer $(35)$. Nevertheless, the potential roles of SKA2 in GC are unknown. The results, 

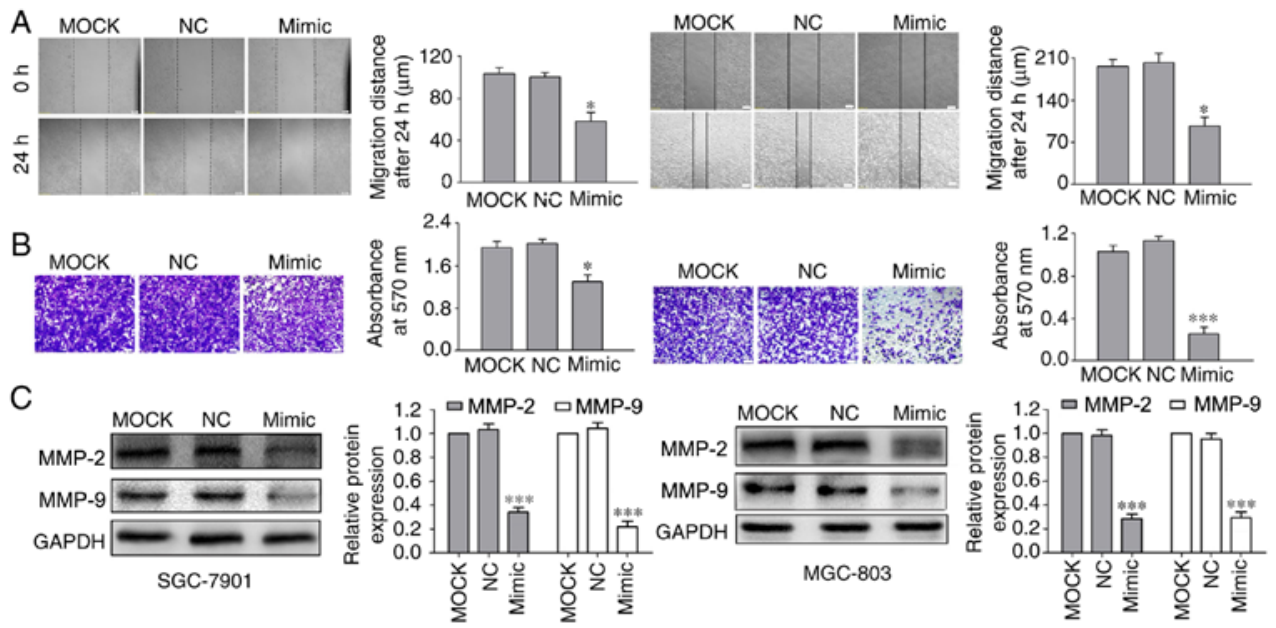

Figure 3. Overexpression of miR-520a-3p inhibits the migratory and invasive capabilities of GC cells. (A) Migratory distance and (B) number of invasive cells in the miR-520a-3p mimics group were significantly decreased compared with the MOCK and NC groups in the wound healing and Transwell invasion assays. ${ }^{*} \mathrm{P}<0.05$ vs. MOCK and NC. (C) Protein expression levels of MMP-2 and MMP-9 were significantly lower in the miR-520a-3p mimics group compared with the MOCK and NC groups. ${ }^{* * *} \mathrm{P}<0.001$ vs. MOCK and NC. Magnification, $\mathrm{x} 100$. Scale bar, $100 \mu \mathrm{m}$. miR, microRNA; GC, gastric cancer; MOCK, mock transfected cells; NC, negative control; MMP, matrix metalloproteinase.

A

\begin{tabular}{|c|c|c|c|}
\hline $\begin{array}{l}\text { Position } 739-746 \text { of SKA2 3'UTR } \\
\text { hsa-miR-520a-3p }\end{array}$ & $5^{\prime}$ & $\begin{array}{r}\text {...AACUGAAAUUAGAAGAGCAC } \\
\text { IIIII } \\
\text { UGUCAGGUUUCCCUUCGUG }\end{array}$ & I UA... \\
\hline SKA2 3'UTR & \multicolumn{2}{|c|}{ 5' ACUGAAAUUAGAAGAGCACUUA } & $3^{\prime}$ \\
\hline miR-520a-3p & \multicolumn{2}{|c|}{ 3' UGUCAGGUUUCCCUUCGUGAAA } & $5^{\prime}$ \\
\hline Mutant 3'UTR & \multicolumn{3}{|c|}{$5^{\prime}$ ACUGAAAUUAGAAGAAGUCGUGAAA $3^{\prime}$} \\
\hline
\end{tabular}

B
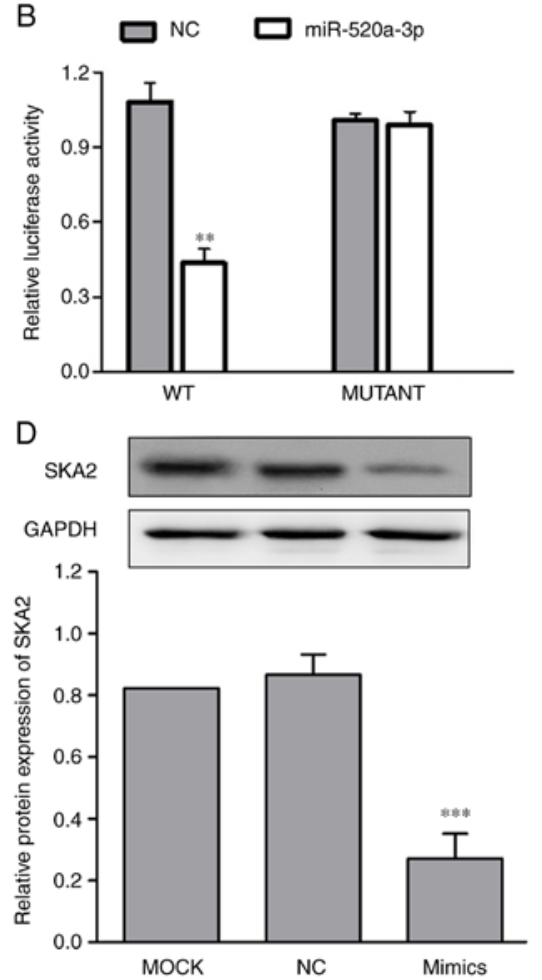

C

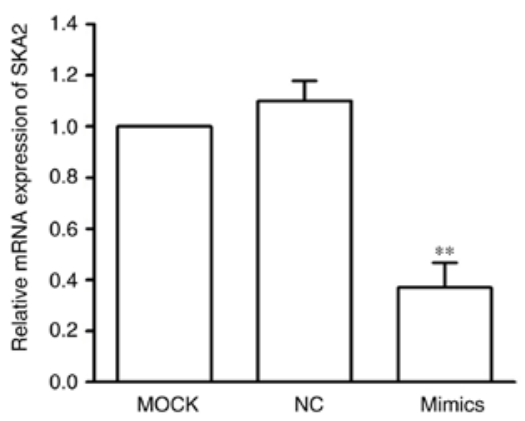

E

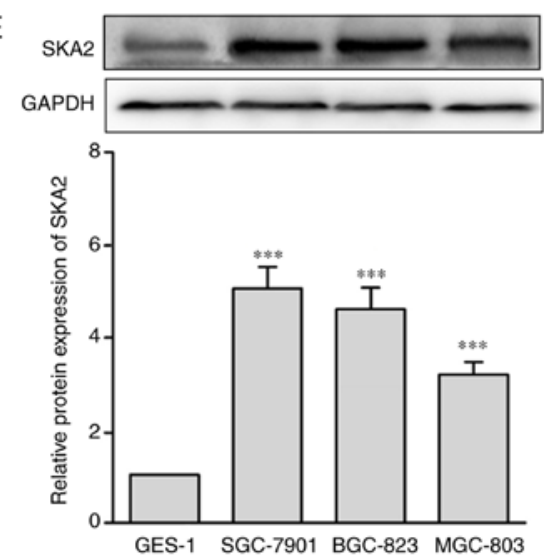

Figure 4. SKA2 is a direct target gene of miR-520a-3p in GC cells. (A) Sequence alignment of miR-520a-3p with 3'UTR of SKA2 predicted by TargetScan and the Mut 3'UTR miR-520a-3p binding sequence. (B) Luciferase activity of SGC-7901 cells was examined via a luciferase reporter assay. ${ }^{* *} \mathrm{P}<0.01 \mathrm{vs}$. mutant. miR-520a-3p decreased SKA2 expression at both the mRNA (C) and protein (D) levels compared to MOCK in SGC-7901 cells. ${ }^{* * *} \mathrm{P}<0.01,{ }^{* * * *} \mathrm{P}<0.001 \mathrm{vs} . \mathrm{MOCK}$ group. (E) SKA2 expression was analyzed in three GC cell lines (SGC-7901, BGC-823 and MGC-803) and a normal gastric epithelial cell line (GES-1). ${ }_{* * * *}^{*}$ P $<0.001$ vs. GES-1. miR, microRNA; GC, gastric cancer; MOCK, mock transfected cells; NC, negative control; WT, wild-type; SKA2, spindle kinetochore-associated protein 2; UTR, untranslated region. 


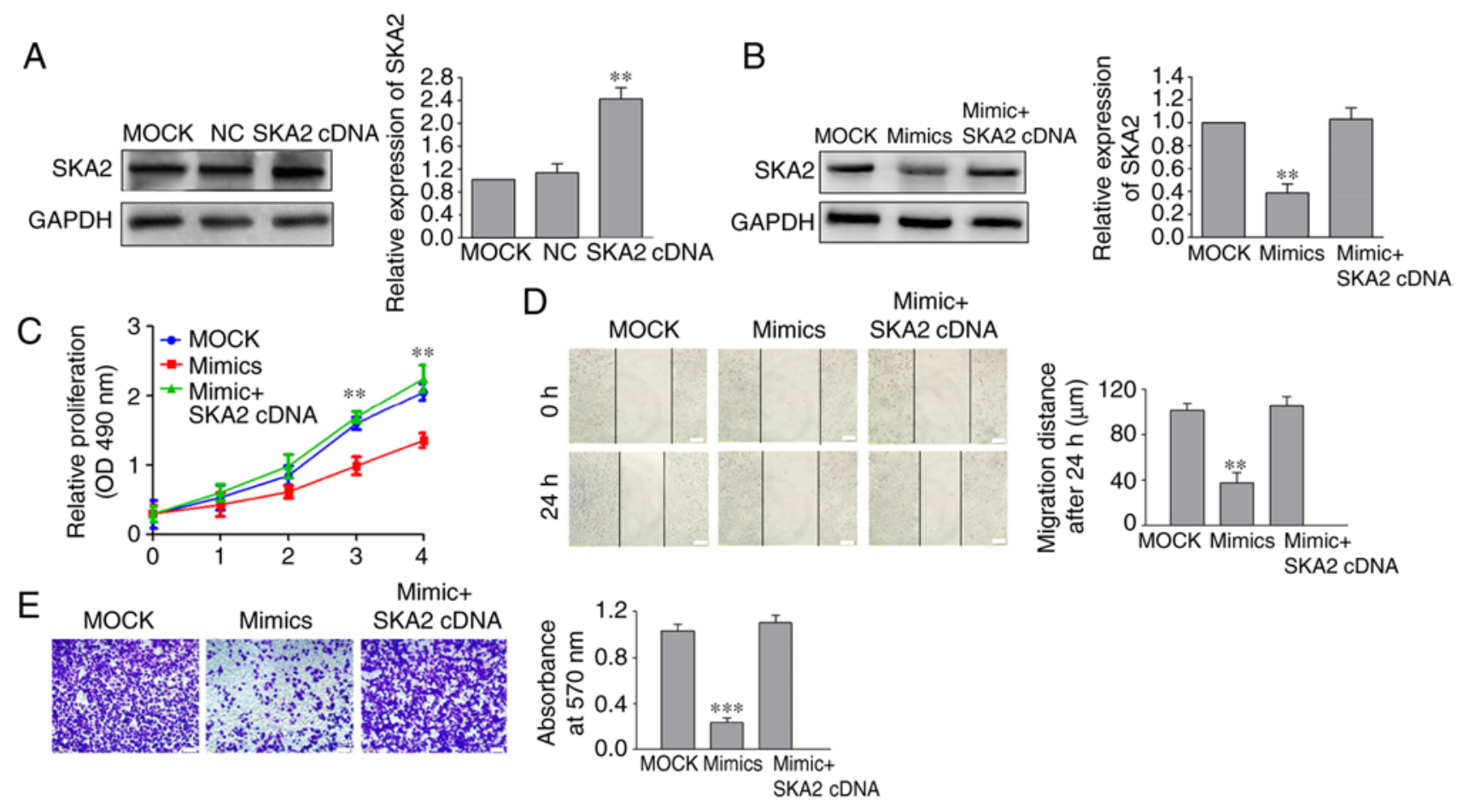

Figure 5. SKA2 regulates miR-520a-3p-mediated inhibition of SGC-7901 cells proliferation, migration and invasion. (A) After cells were transfected with a SKA2 cDNA plasmid, the expression of SKA2 was notably increased in SGC-790 cells. Following co-transfection of miR-520a-3p mimics and SKA2 cDNA into SGC-7901 cells, (B) expression of SKA2 and (C) cell viability were examined by western blotting and cell proliferation assay, respectively. (D) Migration distance and (E) number of invasive cells in GC cells following treatment with miR-520a-3p mimics and SKA2 cDNA were detected by wound healing and Transwell assays, respectively. ${ }^{* *} \mathrm{P}<0.01,{ }^{* * * *} \mathrm{P}<0.001$ vs. the MOCK group. $\mathrm{x} 100$ magnification. Scale bar, $100 \mu \mathrm{m}$. SKA2, spindle kinetochore-associated protein 2; miR, microRNA; GC, gastric cancer; MOCK, mock transfected cells; NC, negative control; OD, optical density.

to the best of our knowledge, are the first to demonstrate that SKA2 was significantly upregulated in gastric cell lines.

As a potential target gene of miR-520a-3p predicted by miRanda and TargetScan software, the present study analyzed the relationship between SKA2 and miR-520a-3p in SGC-7901 cells. The results demonstrated that miR-520a-3p mimics significantly attenuated the mRNA and protein expression of SKA2. Furthermore, downregulation of SKA2 by miR-520a-3p mimics was reversed by SKA 2 cDNA plasmid. These results suggested that miR-520a-3p directly repressed the expression of SKA2 in GC by binding to its 3'UTR.

A previous study reported that miR-520d-3p inhibited GC cell growth and metastatic activity by targeting EphA2 (36). miR-520d-3p and miR-520a-3p are members of the same microRNA family and share a large amount of homology. As they have different sequences and binding sites of target genes, they play a role in tumorigenesis through different regulatory mechanisms. In the present study, the potential value of miRNA-520a-3p and SKA2 as therapeutic targets in cancer diagnosis and targeted therapy was demonstrated.

In summary, miR-520a-3p was downregulated in GC tissues and cell lines. miR-520a-3p also serves a role as a tumor suppressor gene in GC cell lines, decreasing cell proliferation, invasion and migration by targeting SKA2. The novel interaction between miR-520a-3p and SKA2 may contribute to the development of improved targeted therapies for patients with GC. However, due to the complex signaling pathways regulated by microRNAs and the limited sample size, further investigation into the function and regulatory mechanisms of miR-520a-3p in vivo is required. In particular, the expression levels of miR-520a-3p and SKA2 should be determined in a range of cancer types, both in vitro and in vivo, to determine whether the mechanisms underlying miR-520a-3p-mediated oncogenic activity in GC cell lines are replicated elsewhere.

\section{Acknowledgements}

Not applicable.

\section{Funding}

No funding was received.

\section{Availability of data and materials}

All data generated or analyzed during this study are included in this published article.

\section{Authors' contributions}

HS and FR collected and analyzed the data, and wrote and revised the manuscript. XF conceived and designed the experiments. HJ and YC conducted the experiments. All authors read and approved the final manuscript.

\section{Ethics approval and consent to participate}

The present study was approved by The Research Ethics Committee of Ningbo No. 2 Hospital (Zhejiang, China), and all patients provided written informed consent. 


\section{Patient consent for publication}

Not applicable.

\section{Competing interests}

The authors declare that they have no competing interests.

\section{References}

1. Global Burden of Disease Cancer Collaboration; Fitzmaurice C, Allen C, Barber RM, Barregard L, Bhutta ZA, Brenner H, Dicker DJ, Chimed-Orchir O, Dandona R, et al: Global, regional and national cancer incidence, mortality, years of life lost, years lived with disability, and disability-adjusted life-years for 32 cancer groups, 1990 to 2015: A systematic analysis for the global burden of disease study. JAMA Oncol 3: 524-548, 2017.

2. Smyth EC and Cunningham D: Gastric cancer in 2012: Defining treatment standards and novel insights into disease biology. Nat Rev Clin Oncol 10: 73-74, 2013.

3. Zhang J, Song Y, Zhang C, Zhi X, Fu H, Ma Y, Chen Y, Pan F, Wang K, Ni J, et al: Circulating miR-16-5p and miR-19b-3p as two novel potential biomarkers to indicate progression of gastric cancer. Theranostics 5: 733-745, 2015.

4. Jiang $X$ and Wang Z: MiR-16 targets SALL4 to repress the proliferation and migration of gastric cancer. Oncol Lett 16 3005-3012, 2018.

5. Lan H, Lu H, Wang $\mathrm{X}$ and Jin H: MicroRNAs as potential biomarkers in cancer: Opportunities and challenges. Biomed Res Int 2015: 125094, 2015.

6. Valencia-Sanchez MA, Liu J, Hannon GJ and Parker R: Control of translation and mRNA degradation by miRNAs and siRNAs. Genes Dev 20: 515-524, 2006.

7. Luo X, Wang GH, Bian ZL, Li XW, Zhu BY, Jin CJ and Ju SQ Long non-coding RNA CCAL/miR-149/FOXM1 axis promotes metastasis in gastric cancer. Cell Death Dis 9: 993, 2018.

8. Liu J, Wei Y, Li S, Li Y, Liu H, Liu J and Zhu X: MicroRNA-744 promotes cell apoptosis via targeting $\mathrm{B}$ cell lymphoma-2 in gastric cancer cell line SGC-7901. Exp Ther Med 16: 3611-3616, 2018.

9. Yu H, Zhang J, Wen Q, Dai Y, Zhang W, Li F and Li J: MicroRNA-6852 suppresses cell proliferation and invasion via targeting forkhead box J1 in gastric cancer. Exp Ther Med 16 3249-3255, 2018

10. Peng X, Kang Q, Wan R and Wang Z: MiR-26a/HOXC9 dysregulation promotes metastasis and stem cell-like phenotype of gastric cancer. Cell Physiol Biochem 49: 1659-1649, 2018.

11. Yu J, Tan Q, Deng B, Fang C, Qi D and Wang R: The microRNA-520a-3p inhibits proliferation, apoptosis and metastasis by targeting MAP3K2 in non-small cell lung cancer. Am J Cancer Res 5: 802-811, 2015

12. Liu Y, Miao L, Ni R, Zhang H, Li L, Wang X, Li X and Wang J: MicroRNA-520a-3p inhibits proliferation and cancer stem cell phenotype by targeting HOXD8 in non-small cell lung cancer. Oncol Rep 36: 3529-3535, 2016.

13. Lv X, Li CY, Han P and Xu XY: MicroRNA-520a-3p inhibits cell growth and metastasis of non-small cell lung cancer through PI3K/AKT/mTOR signaling pathway. Eur Rev Med Pharmacol Sci 22: 2321-2327, 2018.

14. Li J, Wei J, Mei Z, Yin Y, Li Y, Lu M and Jin S: Suppressing role of miR-520a-3p in breast cancer through CCND1 and CD44. Am J Transl Res 9: 146-154, 2017.

15. Zhang R, Liu R, Liu C, Niu Y, Zhang J, Guo B, Zhang CY, Li J, Yang J and Chen X: A novel role for MiR-520a-3p in regulating EGFR expression in colorectal cancer. Cell Physiol Biochem 42: 1559-1574, 2017.

16. Wang X, Xu Y, Chen X and Xiao J: Dexmedetomidine inhibits osteosarcoma cell proliferation and migration, and promotes apoptosis by regulating MiR-520a-3p. Oncol Res 26: 495-502, 2018.

17. Zhang B, Yu L, Han N, Hu Z, Wang S, Ding L and Jiang J: LINC01116 targets miR-520a-3p and affects IL6R to promote the proliferation and migration of osteosarcoma cells through the Jak-stat signaling pathway. Biomed Pharmacother 107: 270-282, 2018.
18. In H, Solsky I, Palis B, Langdon-Embry M, Ajani J and Sano T: Validation of the 8th edition of the AJCC TNM staging system for gastric cancer using the national cancer database. Ann Surg Oncol 24: 3683-3691, 2017.

19. Livak KJ and Schmittgen TD: Analysis of relative gene expression data using real-time quantitative PCR and the 2(-Delta Delta C(T)) method. Methods 25: 402-408, 2001.

20. Pizon M, Schott DS, Pachmann U and Pachmann K: B7-H3 on circulating epithelial tumor cells correlates with the proliferation marker, $\mathrm{Ki}-67$, and may be associated with the aggressiveness of tumors in breast cancer patients. Int J Oncol 53: 2289-2299, 2018.

21. Krtinic D,Zivadinovic R, Jovic Z, Pesic S, Mihailovic D, Ristic L, Cvetanovic A, Todorovska I, Zivkovic N, Rankovic GN, et al: Significance of the Ki-67 proliferation index in the assessment of the therapeutic response to cisplatin-based chemotherapy in patients with advanced cervical cancer. Eur Rev Med Pharmacol Sci 22: 5149-5155, 2018

22. Komemi O, Epstein Shochet G, Pomeranz M, Fishman A, Pasmanik-Chor M, Drucker L, Tartakover Matalon S and Lishner M: Placenta-conditioned extracellular matrix (ECM) activates breast cancer cell survival mechanisms: A key for future distant metastases. Int J Cancer Sep 144: 1633-1644, 2019.

23. Shay G, Lynch CC and Fingleton B: Moving targets: Emerging roles for MMPs in cancer progression and metastasis. Matrix Biol 44-46: 200-206, 2015.

24. Zhang QH, Qi ST, Wang ZB, Yang CR, Wei YC, Chen L, Ouyang YC, Hou Y, Schatten H and Sun QY: Localization and function of the Ska complex during mouse oocyte meiotic maturation. Cell Cycle 11: 909-916, 2012.

25. Gaitanos TN, Santamaria A, Jeyaprakash AA, Wang B, Conti E and Nigg EA: Stable kinetochore-microtubule interactions depend on the Ska complex and its new component Ska3/C13Orf3. EMBOJ 28: 1442-1452, 2009.

26. Jeyaprakash AA, Santamaria A, Jayachandran U, Chan YW, Benda C, Nigg EA and Conti E: Structural and functional organization of the ska complex, a key component of the kinetochore-microtubule interface. Mol Cell 46: 274-286, 2012.

27. Cao G, Huang B, Liu Z, Zhang J, Xu H, Xia W, Li J, Li S, Chen L, Ding $\mathrm{H}$, et al: Intronic miR-301 feedback regulates its host gene, ska2, in A549 cells by targeting MEOX2 to affect ERK/CREB pathways. Biochem Biophys Res Commun 396: 978-982, 2010.

28. Wang Y, Zhang Y, Zhang C, Weng H, Li Y, Cai W, Xie M, Long Y, Ai Q, Liu Z, et al: The gene pair PRR11 and SKA2 shares a NF-Y-regulated bidirectional promoter and contributes to lung cancer development. Biochim Biophys Acta 1849: 1133-1144, 2015.

29. Wang Y, Weng H, Zhang Y, Long Y, Li Y, Niu Y, Song F and $\mathrm{Bu}$ Y: The PRR11-SKA2 bidirectional transcription unit is negatively regulated by 553 through NF-Y in lung cancer cells. Int J Mol Sci 18: pii: E534, 2017.

30. Shi W, Gerster K, Alajez NM, Tsang J, Waldron L, Pintilie M, Hui AB, Sykes J, P'ng C, Miller N, et al: MicroRNA-301 mediates proliferation and invasion in human breast cancer. Cancer Res 71: 2926-2937, 2011.

31. Ren Z, Yang T, Ding J, Liu W, Meng X, Zhang P, Liu K and Wang P: MiR-520d-3p antitumor activity in human breast cancer via post-transcriptional regulation of spindle and kinetochore associated 2 expression. Am J Transl Res 10: 1097-1108, 2018.

32. Wu H, Wang Y, Chen T, Li Y, Wang H, Zhang L, Chen S, Wang W, Chen C, Yang Q and Chen C: The N-terminal polypeptide derived from vMIP-II exerts its antitumor activity in human breast cancer by regulating lncRNA SPRY4-IT1. Biosci Rep 38: pii: BSR20180411, 2018.

33. Lin CC, Chao PY, Shen CY, Shu JJ, Yen SK, Huang CY and Liu JY: Novel target genes responsive to apoptotic activity by Ocimum gratissimum in human osteosarcoma cells. Am J Chin Med 42: 743-767, 2014.

34. Chang IC, Chiang TI, Lo C, Lai YH, Yue CH, Liu JY, Hsu LS and Lee CJ: Anemone altaica induces apoptosis in human osteosarcoma cells. Am J Chin Med 43: 1031-1042, 2015.

35. Zhuang H, Meng X, Li Y, Wang X, Huang S, Liu K, Hehir M, Fang R, Jiang L, Zhou JX, et al: Cyclic AMP responsive element-binding protein promotes renal cell carcinoma proliferation probably via the expression of spindle and kinetochore-associated protein 2. Oncotarget 7: 16325-16337, 2016.

36. Li R, Yuan W, Mei W, Yang K and Chen Z: MicroRNA 520d-3p inhibits gastric cancer cell proliferation, migration, and invasion by downregulating EphA2 expression. Mol Cell Biochem 396: 295-305, 2014. 